\title{
Efficiently Studying Organic Chemistry
}

written by Eberhard Breitmaier, $1^{\text {st }}$ ed., Thieme: Stuttgart, 2015, e-book, 256 pp, € 39.99, ISBN: 9783132025318

Most text books are designed to accompany one or more specific courses and occasionally may even replace a lecture - if thoroughly worked through. They provide a step-bystep introduction to the given topic and are usually on a high educational level. A much smaller number of text books rather show the characteristics of reference books. They are usually not studied page by page but are consulted when information on a specific topic is needed. Most prominent examples in this field are Jerry March's Advanced Organic Chemistry and a number of books on named reactions. Eberhard Breitmaier (together with Günther Jung) already contributed the text book Organic Chemistry for the German-speaking market, which obviously belongs to the first category. The text book presented here - Efficiently Studying Organic Chemistry - fills a gap, since it neither contains a didactically prepared introduction into the field of organic chemistry, nor a vast amount of usually less important information. It was written to accompany the preparation for an examination in organic chemistry (covering basic courses as given in a bachelor education). It contains (more or less) everything one needs to know in an examination and not much more, which allows focussing on the exam preparation. For this objective it is divided into 81 chapters, each describing a complete topic in organic chemistry (e.g., Reactions of Cycloalkanes and Cycloalkenes; Alcohols; FiveMembered Aromatic Heterocycles; Lipids). Obviously due to this structure it often happens that information necessary for the understanding of an early chapter is only given in a later one. Consequently, this book is only suitable for readers who already have a rough understanding of organic chemistry, for example from a lecture or from a text book.

Efficiently Studying Organic Chemistry is extremely well written. Neither did I notice a single typo, nor any layout mistake in the text or in the schemes. The clear language makes it easy to understand, even for readers not fluent in English. Most of the chemical mistakes I came across are less important: Collins' reagent is not identical with PCC; the unit for the specific rotation is not ${ }^{\circ}$ (though it is most frequently reported in ${ }^{\circ}$ ); the Fischer formulas of the amino acids are incorrect (the carbon with the highest oxidation state should be in the top position); and in the alkaloid chapter on morphine: Morpheus is the god of dreams (while Hypnos is the god of sleep). A vanishingly small fraction of important information is faulty: Page 81: A benzyl halide does not react in an $\mathrm{S}_{\mathrm{N}} 1$ but in an $\mathrm{S}_{\mathrm{N}} 2$ mechanism; Pages 98, 99, 108, 152, 153: Resonance formulas of a given compound must necessarily have the same structure.

The scope of the book is well chosen: Almost all relevant information is given, though often not in the expected chapter (e.g., the Mannich reaction is only given in chapter 63 Benzo-Fused Five-Membered Heterocycles). The only mandatory topic I missed is the influence of $+\mathrm{I}$ (and $-\mathrm{I}$ ) substituents on the electrophilic aromatic substitution (while the influence of $M$ substituents is discussed in detail). Which information should be included and which should be omitted is a very personal decision, but to my feeling the inclusion of numerous test reactions (e.g., Hinsberg separation, Beilstein test, van Slyke test) seems to be rather outdated.

To summarize, Efficiently Studying Organic Chemistry is a very suitable companion in the preparation for an examination in organic chemistry; it is a pleasure to read and its scope is perfectly adapted to the requirements of a typical examination in organic chemistry. I recommend its use especially to those students who benefit from the special features of an e-book.

Joachim Podlech, Karlsruher Institute für Technologie (KIT) 